\title{
जागतिकीकरण आणि दलित समाज
}

\section{Globalization and Dalit society}

\section{Dr. Rakshit Madan Bagde,}

Assistant Professor, Late. Mansaramji Padole Arts College, Ganeshpur Bhandara

ORCID iD - 0000-0002-7507-0244

Web of Science ResearcherID - AAF-2760-2020

SSRN - Author ID: 4770534

Authenticus ID - R-00J-YM2

Vidwan-ID : 221858

सन 1991 च्या मुक्त आर्थिक धोरणाच्या माध्यमातून आलेले जगातिकीकरण मुळात कुणाच्या फायद्याचे ठरणार आहे याचा अजूनही पुर्णपणे आढावा घेण्यात आलेला नाही. देशातील शेती, उद्योग आणि सेवा क्षेत्राचा विकास क्हावा हाच जर हेतू असेल तर आर्थिक कल्याणाचे भारताचे स्वप्न पुर्ण होण्यास वेळ लागणार नाही. परंतू या मुक्त धोरणातून मुठभर लोकांचे कल्याण जोपासले जाणार असेल तर देशातील अन्य जनतेच्या भविष्यावर याचा फार दुरगामी परिणाम होणार आहे. यातून भारत आणि इंडिया संघर्ष हा अटळ राहणार आहे.

\section{- जागतिकीकरण}

डॉ. सी. रंगराजन - “जागतिकीकरण म्हणजे, देशाबाहेरील माहितीप्रवाह, विचार, तंत्रज्ञान, वस्तू, सेवा, भांडवल, वित्त यांचे द्वारे अर्थव्यवस्था आणि समाजाचे एकात्मीकरण होय. यात व्यापार, वित्त, रोजगार, तंत्रज्ञान, दळणवळण, विदेशी स्थलांतर, पर्यावरण, राहणी, शासन, समाजव्यवस्था, संस्कृती अशा सर्व क्षेत्रातून होत राहणारे रूपांतराचा समावेश होय."

- दलित

भारतातील अत्यंत खालच्या स्थरातील लोकांना संबोधला जाणारा शब्द 'दलित' होय. यात परिहास, चांभार, महार आणि भंगी इ. लोकांचा समावेश होतो आणि यांनाच अस्पृश्य असेही संबोधले जाते.

- जागतिकीकरणाचे धोरण

भारतात सन 1991 पासून जागतिकीकरणाला सुरुवात झाली. परंतु 1995 पासून जागतिकीकरणाचा वेग वाढविण्यात आला आहे. अर्थव्यवस्थेचे जागतिकीकरण महणजे स्पर्धेच्या माध्यमाद्वारे देशांतर्गत किंमतीचे आंतरराष्ट्रीय स्तरावरील किंमतीशी समानीकरण करणे होय आणि हचाकरिता साधनांचा मुक्तपणे संचार होऊ देणे होय.

जागतिक अधिकोषाने जागतिकीकरणाचे वर्णन पुढीलप्रमाणे केले आहे - 
1) उपभोग्य वस्तुसह सर्व वस्तुंच्या आयातीवरील निर्बंध कमी करणे.

2) आयात दर कमी पातळीवर आणणे.

3) सार्वजनिक उद्योगांचे खाजगीकरण करणे .

म्हणजेच 'जागतिकीकरण एक स्पर्धा आणि नवे तंत्रज्ञान यातून उत्पादकता आणि उत्पादन वाढविणे होय.'

भारतीय अर्थव्यवस्थेच्या जागतिकीकरणात -

1) उपभोग्य वस्तुंच्या आयातीवरील निर्बंध उठविणे .

2) जकात प्रशुल्क कमी करणे .

3) विदेशी गुंतवणुकीला पूर्ण मुभा देणे.

4) रूपया परिवर्तनशील करणे .

5) अधिकोष आणि विमा क्षेत्र खुले करणे .

इ. बाबींचा समावेश होतो .

समय, अंतर आणि मूल्य हचा तीन महत्त्वाच्या आयामांच्या दृष्टिकोनातून जागतिकीकरणाचा विचार केल्या जातो. त्यामुळेच "जागतिकीकरण ही एक मिश्र संकल्पना असून त्याचा प्रभाव फार दुरवर पसरलेला आहे. हे आश्चर्य नसून त्याचे अनेक मनोभावीक अनुमान लावण्यात आले आहेत. हा विषय राजकारणात ताजा आहे, जागतिकीकरणाचा उपयोग जगातील लोकांना आर्थिक संपन्नता प्राप्त करण्यासाठी केला जातो. तर एकीकडे यावर असाही दोष लावला जातो की, यामुळे अनेक नवीन प्रश्न निर्माण होत आहेत."

\section{- उद्देश}

अध्ययनातील महत्वाचे उद्देश जागतिकीकरणाचा दलितांच्या आर्थिक तसेच सामाजीक विकासावर कोणता परिणाम झालेला आहे याचा आढावा घेणे हा आहे. त्याकरीता त्यांच्या निवास, शिक्षण, आरोग्य, रोजगार, सामाजिक सुरक्षा इ. मुद्यांचा विचार केला जाणार आहे.

- संशोधन पद्धती 
सदर अध्ययनाकरीता द्वितीय माहीती स्त्रोतांचा आधार घेण्यात येणार आहे. यात संशोधनपर लेख, पुस्तके आणि अनेक वार्षीक अहवालांचा उपयोग होणार आहे.

\section{• संशोधन साहित्याचा आढावा}

जॉन सि. बि. वेबस्टार (2001) सदर संशोधपर लेखात दलितांच्या राजकिय, आर्थिक, सामाजिक आणि धार्मिक बाजूचा आढावा घेतलेला आहे. वेबस्टार च्या मते, दलितांचे होणारे शोषण हे त्यांना ख्विश्चन धर्माकडे आकर्षीत करीत आहे. आणि त्यामुळे भारतातील ख्रिश्चनांच्या संख्येत होणारी वाढ ही दलितांच्या धर्म परिवर्तनामुळे होत आहे.

Ellyn Artis, Chad Doobay and Karen Lyons सदर संशोधनात गुजरातमधील दलितांच्या प्राथमीक शिक्षणाचे अध्ययन केलेले आहे. ज्यात संशोधकांनी सरकारकडे काही महत्वपुर्ण अशा सिफारशी केलेल्या आहेत. ज्यात, सरकारने दर्जेदार शिक्षण पुरविणे, शिक्षकांचा दर्जा उंचावा म्हणून प्रशिक्षणाची सोय कारणे, शिक्षकांना मानवाधिकाचे धडे देऊन वर्गात समानतेचे वातावरण निर्माण करावे आणि विद्यार्थाचे अधिकाराचे हनन करणारे शिक्षकावर योग्य कार्यवाही करणे.

Dr. Anand Teltumbde संशोधनपर लेखात अन्नसुरक्षा, तेजी, रोजगार आणि गरिबी या आधारे दलितांवरील परिणामाची चर्चा केलेली आहे. यातील निष्कर्षाच्या आधारे संशोधकानी दलितांच्या गरिबीचे प्रमाण सन 1996 पासून वाढत चाललेले असल्याचे स्पष्ट केले आहे. नोकयांमध्ये दलितांचे प्रमाण वाढविण्यात यावे यावर तेलतुंबडे भर देतात.

सन 2011 च्या जनगणनेनुसार भारतात $16.2 \%$ म्हणजे $16,66,35,700$ एवढी संख्या अनुसूचित जारींची तर $8.2 \%$ म्हणजे $8,43,26,240$ लोकसंख्या जनजातींची आहे. ज्यातील अनुसूचित जार्तींची $28.29 \%$ संख्या पंजाब मधील आहे तर सर्वात कमी म्हणजे $0.03 \%$ मिजोरम मधील आहे.

देशाचा सन 1991 पासून आढावा घेतल्यास असे प्रामुख्याने लक्षात येते की, देशात 6 लाख गावांपर्यत अजूनही पिण्याचे स्वच्छ पाणी पोहचलेले नाही त्यामुळे दुषीत पाण्यामुळे 10 लाख बालके दरवर्षी मरत आहेत. देशातील 30 कोटी लोकांपर्यंत अजूनही अन्न पोहचलेले नाही याच्या परिणामस्वसूप 29 लाख बालके दरवर्षी कुपोषणाचे बळी ठरत आहेत. अक्षम आरोग्याच्या सोयीमूळे 5 लाख लोक दरवर्षी क्षय रोगाने मरत आहेत तर मलेरीयाने 20 लाख लोकांचा बळी जात आहे. जगातले $94 \%$ कृष्ठ रोगी भारतात दिसून येतात. $80 \%$ भारतीय महिला या रक्तीतील हिमोग्लोबिनच्या कमतरतेने ग्रासलेल्या आहेत. असे हे भारताचे भयानक चित्र जागतिकीकरणाच्या काळात निर्माण झालेले आहे. 
देशातील जनसमूदायाचा विकास झाला की नाही याची गणना त्या लोकांकरीता रोजगार, अन्न, आरोग्य, शिक्षण, निवारा आणि सामाजिक सुरक्षा मुबलक प्रमाणात उपलब्ध करण्यात आले की नाही यावरून ठरविण्यात येत असते. या सर्व उत्कर्षाच्या काळात देशाच्या विकासाच्या मुख्य प्रवाहातून देशातील मोठा जनसमुदाय मागे पडलेला दिसून येतो, ज्यात बहुसंख्य अनु. जाती आणि जनजातींची संख्या समाविष्ट आहे. देशात कितीही औद्योगिकरणाची लाट आली तरी वास्तविकता ही आहे की भारत अजुनही कृषीप्रधान देश आहे. यातील $70 \%$ जनता आजही शेतीव्यवसायाशी सबंधीत आहे. अनु. जातीचा विचार करता आजही $72.93 \%$ लोक शेतीव्यवसायाशी सबंधीत असलेले दिसून येतात. यात $10 \%$ शेतमजुर असून त्यांच्याकडे शेतीचा एक तुकडाही नाही. $65 \%$ लोकांकडे सरासरी 0.40 हेक्टर जमीनीच्या तूकड्याचे मालक आहेत. यातील फक्त $1.10 \%$ लोकांकडेच जमीनीचे मोठे तुकडे दिसून येतात. याचाच परिणाम म्हणजे हल्ली $51 \%$ अनु. जातींच्या लोकांना शेतमजुर म्हणून काम करावे लागत आहे. येणाया काळात ही संख्या $65 \%$ च्याही वर जाण्याची शक्यता आहे.

सन 2003 मध्ये अनु. जातींचे फक्त $16.52 \%$ लोकच शासकीय सेवेमध्ये समाविष्ट होते. शिक्षणाचा आढावा घेता $54.70 \%$ अनु. जातींमध्ये शिक्षणाचे प्रमाण दिसून येते. यातील फक्त $4.4 \%$ पदव्युत्तर किंवा त्यापेक्षा जास्त शिकलेले आहे. यात महिलांचे प्रमाण मात्र अत्यंत कमी असून ते $1 \%$ आहे. $80.5 \%$ अनु. जातीच्या लोकांचे घर आजही कच्च्या स्वरूपाचे आहे. ज्यातील फक्त $44.3 \%$ लोकांच्या घरी विद्युत जोडणी पोहचलेली आहे. सन 2001 मधील आकडेवारी नुसार $23.7 \%$ लोकांच्या घरीच संडासाची सुविधा होती. यातील विस्मयकारक बाब म्हणजे देशात चाललेला गरिबी निर्मूलनाचा योग्य तो परिणाम अजूनही अनु. जातींच्या लोकांवर दिसून येत नाही कारण आज घडीला अनु. जातीच्या लोकांची ग्रामीण गरिबी $36.2 \%$ अस्तित्वात असलेली दिसून येते आहे.

\section{दलितांची वास्तविक स्थिती सन 2001 ते 2005 पर्यंत}

> $37.8 \%$ गावात दलित विद्यार्थांना सरकारी शाळेत वर्गाबाहेर बसविले जाते.

$>27.6 \%$ गावात दलितांना पोलीस ठाण्यात जाण्याकरीता अडविले जाते.

> $25.7 \%$ गावात दलितांना सरकारी रेशन दूकानात प्रवेश करण्याला मनाही केली जाते.

> $33 \%$ गावात दलितांच्या घरात सरकारी आरोग्य सेवक प्रवेश करीत नाही.

$>23.5 \%$ गावात दलितांच्या घरी पोस्टमन पत्र टाकत नाही.

$>14.4 \%$ गावात दलितांना पंचायती मध्ये प्रवेश नाही.

$>12 \%$ गावात दलितांना मतदानाकरीता वेगळया रांगेत उभे राहावे लागते.

$>48.4 \%$ गावात दलितांना पिण्याच्या पाण्याची सोय नाही.

> $35 \%$ गावात दलितांना बाजारात वस्तू विकण्यास मनाही केली जाते.

> $47 \%$ गावात दलितांना सहकारी दुग्ध संस्थांना दुग्ध विकण्यास मनाई केली जाते.

$>25 \%$ गावात दलितांना दुग्ध खरेदी करण्यास मनाई केली जाते. 
$>25 \%$ गावात दलितांना अत्यंत कमी दराने मजूरी दिली जाते.

$>37 \%$ गावात दलित कामगारांना श्रमाची कमी मजूरी दिली जाते.

$>64 \%$ दलित लोकांना अजूनही मंदीरात प्रवेश मिळत नाही.

> $50 \%$ गावात दलितांना स्मशानभूमी पर्यत पोहचू दिल्या जात नाही.

$>73 \%$ गावात दलितांना बिगर दलितांच्या घरी प्रवेश नाही.

$>70 \%$ गावातील दलितांना बिगर दलितांसोबत बसून जेवन करू दिल्या जात नाही.

$>35.8 \%$ दलितांना गावातील रेशन दूकानातून खरेदी करू दिल्या जात नाही.

दलितांनावरील अत्याचार सन 2001-2005
* दररोज 27 Atrocities चे गुन्हे होत आहेत.
* दर आठवडयाला 13 दलितांची हत्या होते आहे.
* दर आठवडयाला 5 दलितांची घरे जाळली जातात.
* दर आठवडयाला 6 दलितांचे अपहरण केले जाते आहे.
* दरदिवशी 3 दलित महीलेवर बलात्कार होतो आहे.
* दरदिवशी 11 दलितांवर मारपीट केली जाते.
* दरमिनिटाला 18 दलितांवर अन्याय होतो आहे.

सन 2002 पर्यंत 7,099 or $18.70 \%$ दलितांच्या शोषणाचे गुन्हे पोलीसांकडून नोंदविल्या गेले नाहीत. याच काळात $1,26,009$ or $77.69 \%$ गुन्हे न्यायालयासामोर आले. त्यापैकी फक्त $21.72 \%$ गुन्यांचाच निकाल समोर आला. Indian Police Statistics च्या रिपोर्टनुसार $23.9 \%$ गुन्हयांचा अजूनही शोध लागलेला नाही. 2005 च्या शेवटपर्यंत $80.2 \%$ दलितांच्या शोषणाचे गुन्हे न्यायालयात स्थगीत होते.

$>79.8 \%$ दलित आजही ग्रामीण भारतात राहतात.

द दलितांमध्ये दररोज 100 मुलांचा जन्म होतो त्यापैकी 12 मुले आपल्या पाचव्या वाढदिवसा पर्यंतही जगू शकत नाही.

$>$ दलितांमधील 5 मुलांपैकी फक्त 1 मूल शाळा शिकून बाहेर पडते.

$>$ आजही 24.4 लाख दलित कामगार गुलामीत काम करतात.

- निष्कर्ष

उच्च शिक्षणातील सबसिडी कमी करा असा जागतिक बँँेचा आदेश आहे . शिक्षणाचा सेवा व्यापारात समावेश करून 'शिक्षणाचा व्यापार' सुरू झाला आहे. पूर्वी मागासवर्गीयांना शिक्षणाचा अधिकार नव्हता. आता अधिकार असूनही खाजगी शिक्षण घेता येत नाही. त्यामुळे या विषमव्यवस्थेत उच्चवर्णीयांचाच फायदा होणार आहे. मागासवर्गीय समाजाने मोठचा कष्टाने मिळविलेले 'आरक्षण' खाजगीकरणाच्या माध्यमातून संपुष्टात येत आहे . 
राज्यघटनेतील मार्गदर्शक तत्वानुसार वय 6 ते 14 वर्षाखालील मुलांना मोफत शिक्षण मिळाले पाहिजे हे तत्त्व 52 वर्षानंतर मान्य करण्यात आले. स्पर्धेच्या युगात केवळ अक्षर ओळख पुरेशी ठरणार नाही, महणूनच इंग्रजी माध्यमाच्या शाळा खाजगी क्षेत्रात उदयास आल्या. हचा इंग्रजी माध्यमाच्या शाळांची फी जास्त असल्यामुळे गरीबांच्या मुलांना इंग्रजी अक्षर ओळखणे कठीण जात आहे.

मुक्त आर्थिक धोरणातून उच्च शिक्षण खुल्या बाजारात येत आहे. उद्योगपती शिक्षणात हस्तक्षेप करीत आहेत. त्यामुळे शिक्षणावर विपरित परिणाम होऊन उच्च शिक्षणाचा दर्जा खालावत आहे. माध्यमिक शिक्षणाकरिता सरकारने इंग्रजी शाळा सुरू कराव्यात कारण गरीबांना इंग्रजी शिक्षण महाग होत आहे .

वैद्यकीय सेवेच्या खाजगीकरणामुळे गरीब जनतेला चांगले आरोग्य अशक्य बाब बनली आहे. विदेशी कंपन्यांना 100 टक्के गुंतवणुकीत मुभा दिल्याने त्याचा गरीबांच्या आरोग्यावर विपरित परिणाम होणार आहे .

सन 2002-03 च्या अर्थसंकल्पात 42,000 कामगारांना स्वेच्छा निवृत्तीच्या नावावर कमी करण्याचा संकल्प सरकारने मांडला होता. महाराष्ट्र सरकारने याच्याही पुढे जाऊन 2003 च्या अर्थसंकल्पात 29,828 कर्मचाय्यांना अतिरिक्त ठरविले. 34 वेगवेगळचा विभागांचा आढावा घेऊन 60 हजारांवर कर्मचान्यांना अतिरिक्त महणून घोषित करण्यात आले .

डॉ. आंबेडकरांनी शेतीच्या राष्ट्रीयीकरणावर भर दिला होता. परंतु मुक्त आर्थिक धोरणात शेतीचे खाजगीकरण करण्यात येऊन शेतीला कोणत्याही सोयी सुविधा सरकार देवू इच्छित नाही. सरकारने आदिवासींच्या जमिनी विक्रीला काढण्याचे नवे धोरण सुरू केले आहे. तसेच 'सेझ' च्या माध्यमातून सुपीक जमिनी मोठचा कंपन्यांना मातीमोल भावाने विकल्या जात आहेत .

"नवीन आर्थिक धोरणापासून अपेक्षित असलेले फायदे पंधरा कोटी दलितांपर्यंत पोहचले नाहीत. प्रशासनाने मंजूर केलेला निधी दलितांसारख्या वंचित घटकापर्यंत पोहचल्याचा सबळ पुरावा अद्याप हाती आलेला नाही." विलास दासगुप्ता (प्राध्यापक, कलकत्ता विद्यापीठ)

संदर्भ ग्रंथ -

कांबळे उत्तम (2002) - जागतिकीकरण आणि दलितांचे प्रश्न, सुगावा प्रकाशन पुणे.

थोरात सुखदेव (2009) दलित इन इंडिया, सेज प्रकाशन, दिल्ली. 
बागडे रक्षित मदन (2008) "मुक्त आर्थिक धोरणामूळे निर्माण होणाय्या समस्यांचे डॉ. आंबेडकरांच्या आर्थिक तत्त्वज्ञानातून चिकित्सक विश्लेषण" लघू शोध प्रबंध, डॉ. आंबेडकर विचारधारा विभाग, रा.तु.म. नागपूर विद्यापिठ. DALITS OF INDIA (2006) - WWW.IDSN.ORG - IDSN (INTERNATIONAL DALIT SOLIDARITY NETWORK) NøRREBROGADE 66C, 1.SAL2200, DENMARK. 